\title{
Optimizing Scan Homogeneity for Building Full-3D Lidars based on Rotating a Multi-Beam Velodyne Rangefinder*
}

\author{
Anthony Mandow ${ }^{1}$, Jesús Morales ${ }^{1}$, Jose Antonio Gomez-Ruiz ${ }^{1}$ and Alfonso J. García-Cerezo ${ }^{1}$
}

\begin{abstract}
Multi-beam lidar (MBL) scanners are compact, light, and accessible 3D sensors with high data rates, but they offer limited vertical resolution and field of view (FOV). Some recent robotics research has profited from the addition of a degree-of-freedom (DOF) to an MBL to build rotating multibeam lidars (RMBL) that can achieve high-resolution scans with full spherical FOV. In a previous work, we offered a methodology to analyze the complex 3D scan measurement distributions produced by RMBLs with a rolling DOF and no pitching. In this paper, we investigate the effect of introducing constant pitch angles in the construction of the RMBLs with the purpose of finding a kinematic configuration that optimizes scan homogeneity with a spherical FOV. To this end, we propose a scalar index of 3D sensor homogeneity that is based on the spherical formulation of Ripley's K function. The optimization is performed for the widely used Puck (VLP-16) and HDL-32 sensors by Velodyne.
\end{abstract}

\section{INTRODUCTION}

In the last few years, multi-beam lidar (MBL) rangefinders, like those developed by Velodyne, are becoming increasingly applied in robotic vehicles, such as in urban scene understanding [1], emergency response robots [12], off-road ground vehicles [8], and mobile manipulators [2]. These sensors can be considered as a hybrid between 2D and 3D scanners, as they consist on a spinning structure that holds a number of independent laser transceivers to scan planes with different elevation angles within a fixed vertical field of view (FOV).

With the decreasing cost of MBL sensors [14], rotating multi-beam lidars (RMBL) built by adding a degree of freedom (DOF) to a commercial MBL may arguably become a common solution to obtain affordable rapid full-3D high resolution scans in the close future. This idea is supported by recent examples reported in the literature. The first solutions incorporated Velodyne HDL-64E lidars on tilting (or nodding) mechanisms for robotic mapping vehicles in mines [7] and tunnels [3]. Lighter MBL devices, like the Velodyne Puck, have favored more compact tilting systems [8][5]. Furthermore, a complete spherical FOV can be achieved by mounting the MBL on a continuous rotation mechanism [6][2].

In contrast to rotating single-beam 2D lidars, whose nonhomogeneous measurement distributions can be characterized by constant vertical and horizontal angular resolutions

\footnotetext{
*This work was partially funded by the Spanish project DPI2015-65186R. The publication has received support from Universidad de Málaga, Campus de Excelencia Andalucía Tech.

${ }^{1}$ Robotics and Mechatronics Lab, Andalucía Tech, Universidad de Málaga, 29071 Málaga, Spain \{amandow, jesus.morales, janto, ajgarcia\}@uma.es
}

[16], overlapping beams in RMBLs provoke much more complex scan patterns. Understanding these patterns is crucial for effectively building and applying customized 3D sensors. In a recent work [5], we proposed a methodology based on a spherical formulation of Ripley's $K$ function [10] to analyze the distribution of 3D range measurements projected on a hollow sphere. This methodology was applied to an RMBL configuration where the vertical axis of the constituent MBL is perpendicular to the rotation axis (i.e., zero pitch angle), as in [7][3][8][2]. However, as indicated by Neumann et al. [6], rotating a Velodyne Puck with a constant pitch inclination can improve measurement density distribution.

In this paper, we investigate the effect of pitch angles in RMBL measurement distributions in order to find a kinematic configuration that optimizes scan homogeneity and with a full-sphere FOV. With this purpose, the originality of this work with respect to [5] is threefold: $(i)$ we consider a more general build configuration where the Velodyne sensor is not perpendicular to the axis of the rolling DOF but can have a constant pitch angle; (ii) we define a new scalar index to assess the homogeneity of 3D sensor data distribution based on the spherical formulation of Ripley's $K$ function; and (iii) we optimize the pitch angle that maximizes the homogeneity for RMBLs based on the Puck VLP-16 and the HDL-32.

The rest of the paper is organized as follows. Section II defines a general RMBL that includes a pitch angle. Section III reviews the application of the spherical extension of Ripley's $K$ function to analyze 3D scan measurement distribution and proposes a scalar homogeneity index. Section IV analyzes the effect of the pitch angle and discusses optimal configurations for rotating the Puck and the HDL-32 rangefinders. Finally, Section V offers the conclusions.

\section{GENERAL RMBL DEFINITION}

Let us define the local frame $X_{v} Y_{v} Z_{v}$ of an MBL with its origin in the optical center, its $Y_{v}$ axis in the forward direction and $Z_{v}$ pointing upwards. This is illustrated in Fig. 1 for the case of the Puck sensor. An MBL scans points in spherical coordinates $(R, \omega, \alpha)$, which correspond to Cartesian coordinates $\left(x_{v}, y_{v}, z_{v}\right)$ for each measured point:

$$
\begin{aligned}
& x_{v}=R \cos (\omega) \sin (\alpha), \\
& y_{v}=R \cos (\omega) \cos (\alpha), \\
& z_{v}=R \sin (\omega) .
\end{aligned}
$$

The local frame $X Y Z$ of the RMBL resulting from the addition of a rotating mechanism to the MBL is illustrated in 

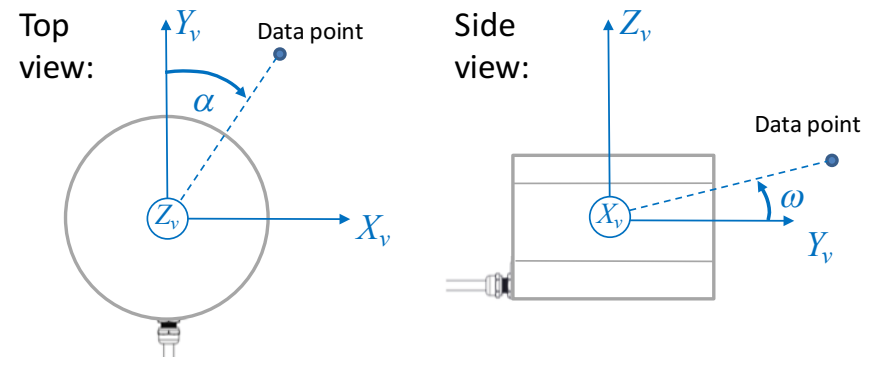

Fig. 1. Local frame for a MBL, illustrated for a Puck sensor.

Fig. 2. Let us consider that the rotation axis is parallel to one of the MBL axes; in this case, without loss of generality, the rotation axis is considered to be $Y$. Then, when the rotation angle $\gamma$ is null, $X_{v}$ is parallel to $X$. The case of RMBL sensors where the vertical axis of the constituent MBL (i.e., $\left.Z_{v}\right)$ is perpendicular to the rotation axis [7][3][8][2] was considered in [5] and is represented as $X_{v} Y_{v}^{\prime} Z_{v}^{\prime}$ in Fig. 2. In this work, we consider a more general configuration where the MBL can be installed with a constant pitch angle $\beta$ about its $X_{v}$, which is the angle between $Z_{v}$ and the $X Z$ plane. Furthermore, in practice, the rotation axis $Y$ can be at some small constant distance $d$ from the MBL origin.

With this frame definition, the computation of Cartesian coordinates $(x, y, z)$ for a point in the frame of an RMBL with kinematic parameters $d$ and $\beta$ from a MBL measurement $(R, \omega, \alpha)$ is as follows:

$$
\begin{aligned}
x & =R \cos (\omega+\beta) \sin (\alpha) \cos (\gamma) \\
& +R \sin (\omega+\beta) \sin (\gamma)+d \sin (\gamma), \\
y & =R \cos (\omega+\beta) \cos (\alpha), \\
z & =R \sin (\omega+\beta) \cos (\gamma) \\
& -R \cos (\omega+\beta) \sin (\alpha) \sin (\gamma)+d \cos (\gamma) .
\end{aligned}
$$

\section{HOMOGENEITY ASSESSMENT OF 3D SCAN DATA}

This section reviews the application of the spherical extension of Ripley's $K$ function to analyze 3D scan measurement distribution. Based on this formulation, we propose a scalar index to evaluate data homogeneity.

\section{A. Spherical 3D Scan Data Analysis with the K Function}

The original Ripley's $K$ function [9] is a spatial statistics tool for analyzing data homogeneity of points in the plane. A set of points is considered homogeneous if the same number of points occurs in any circular region of a given area. The comparison between the $K$ function for the point set and the $K$ function for complete spatial randomness (CSR), denoted as $K_{c s r}$, allows determining whether points have a random, dispersed or clustered distribution over a range of distances [13].

In particular, a positive value of the difference $\left(K-K_{c s r}\right)$ for a given distance indicates clustering, i.e., that the average number of neighbor points for that particular range of distances is higher than the average for the whole distribution, whereas negative values indicate dispersion.

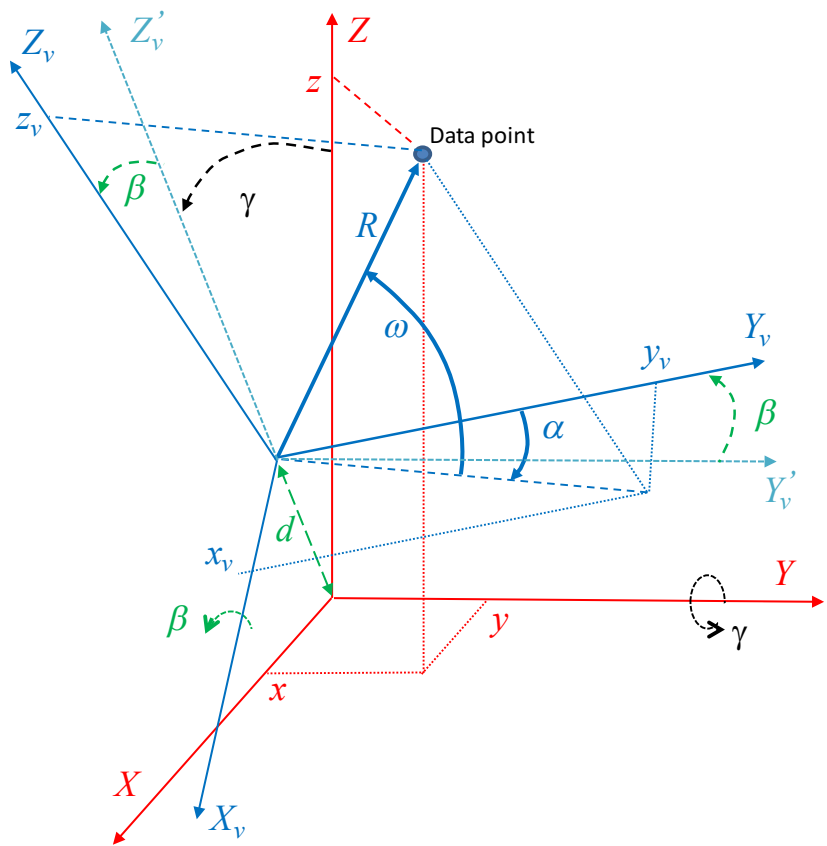

Fig. 2. Frames for a generalized RMBL sensor. The MBL frame is depicted in blue; the RMBL frame is in red; the additional DOF $\gamma$ is in black; constant kinematic parameters $d$ and $\beta$ are in green.

Simulating measured points from the center of a hollow sphere is a common approach to evaluate 3D scan data density distributions [16][4][11]. Because of this, using a variation of the $K$ function for spherical surfaces [10] is proposed in [5]. The $K$ function for spherical CSR is given by:

$$
K_{c s r}(\rho)=2 \pi R^{2}(1-\cos (\rho))
$$

where $R$ is the radius of the sphere and $\rho=r / R$ represents great-circle distance $r$ normalized in the $[0, \pi]$ interval.

Given a set of $n$ sphere points $p_{1}, \ldots, p_{n}$ the estimation of the $K$ function for points on a sphere can be computed as:

$$
\hat{K}(\rho)=\frac{8 \pi R^{2}}{n(n-1)} \sum_{i=1}^{n} \sum_{j=i+1}^{n} I\left(\theta\left(p_{i}, p_{j}\right) \leq \rho\right),
$$

where $\theta\left(p_{i}, p_{j}\right)$ represents the angle corresponding to the great-circle distance between $p_{i}$ and $p_{j}$, and $I(\cdot)$ is the indicator function.

\section{B. Definition of a Scalar Homogeneity Index}

The difference $\hat{K}(\rho)-K_{c s r}(\rho)$ computed from (8) and (7) results in a curve that allows analyzing the point distribution for all normalized great circle distances $\rho$.

However, a scalar cost function that indicates overall homogeneity is required to optimize the kinematic parameter $\beta$ of a customized RMBL configuration. With this purpose, let us define index $\eta$ for a given pitch angle $\beta$ as:

$$
\eta(\beta)=\int_{0}^{\pi}\left|\hat{K}(\rho ; \beta)-K_{c s r}(\rho)\right| d \rho
$$


which weighs clustering and dispersion equally. This index is a measure of inhomogeneity that has to be minimized to optimize homogeneity in 3D scan measurement distributions.

\section{ANALYSIS AND OPTIMIZATION OF THE PITCH ANGLE FOR VELODYNE-BASED RMBLS}

\section{A. Velodyne sensors}

In this analysis we consider two representative examples of commercial multi-beam sensors usually found in robotics applications: the VPL-16 Puck and HDL-32 by Velodyne. The manufacturer specifications for these MBLs are summarized in Table I. The major differences between these two sensors concerning scan data distribution lie in the number of laser transceivers and the vertical FOV. The Puck has 16 individual laser/detectors arranged in a $30^{\circ} \mathrm{FOV}$, which yields a vertical resolution of $2.0^{\circ}$, whereas the HDL-32 has 32 transducers within a FOV of $40^{\circ}$ with a vertical resolution of $1.29^{\circ}$. Furthermore, the Puck FOV is symmetrical with respect to its horizontal plane, whereas the HDL-32 has an asymmetrical FOV with a downward shift.

\section{B. Hollow Sphere RMBL Sampling}

Hollow sphere measurements have been obtained by simulating a full rotation of the roll angle from $\gamma=0^{\circ}$ to $\gamma=360^{\circ}$. Cartesian points have been computed with (4)-(6) and $R=10 \mathrm{~m}$. For the sake of simplicity, it will be assumed that the sphere radius is large enough to make the deviation between the center of the sphere (i.e., the RMBL's origin) and its optical center negligible (i.e., $d \approx 0$ ). Besides, for generalization, no shadows or other FOV limitations due to a particular mechanism are considered. The scan resolution is determined by the angular velocity of $\gamma$.

Fig. 3 illustrates the resulting data distribution for an RMBL based on the Puck sensor, with a rotation speed of $d \gamma / d t=300^{\circ} / \mathrm{s}$, for four different values of pitch angle $\beta$. From a qualitative standpoint, the case of an aligned sensor (i.e., $\beta=0$ ) shown in Fig. 3(a) has focal points with a higher density in the rotation axis as well as distinctive stripes of unsampled spots [5]. As the Puck FOV is symmetrical, $\beta=0$ makes that the center of this FOV is aligned with the

TABLE I

MANUfACTURER SPECIFICATIONS FOR THE PUCK AND HDL-32 SENSORS [15].

\begin{tabular}{lll}
\hline & Puck (VLP-16) & HDL-32 \\
\hline Channels & 16 & 32 \\
Range & $1 \mathrm{~m}$ to $100 \mathrm{~m}$ & $1 \mathrm{~m}$ to $70 \mathrm{~m}$ \\
Accuracy & $\pm 3 \mathrm{~cm}$ & $\pm 2 \mathrm{~cm}$ \\
Data & Distance / & Distance / \\
& Calibrated reflectivities & Calibrated reflectivities \\
Data Rate & 300,000 points/s & 700,000 points $/ \mathrm{s}$ \\
Vertical FOV & $30^{\circ}:\left[-15^{\circ},+15^{\circ}\right]$ & $40^{\circ}:\left[-30^{\circ},+10^{\circ}\right]$ \\
Vertical Res. & $2.0^{\circ}$ & $1.29^{\circ}$ \\
Horiz. FOV & $360^{\circ}$ & $360^{\circ}$ \\
Horiz. Res. & $0.1^{\circ}$ to $0.4^{\circ}$ (progr.) & $0.08^{\circ}$ to $0.35^{\circ}$ (progr.) \\
Rotation Rate & $5 \mathrm{~Hz}$ to $20 \mathrm{~Hz}$ & $5 \mathrm{~Hz}$ to $20 \mathrm{~Hz}$ \\
Size & $103 \mathrm{~mm} 72 \mathrm{~mm}$ & $85.3 \mathrm{~mm} \times 149.9 \mathrm{~mm}$ \\
Weight & $0.83 \mathrm{Kg}$ & $1.3 \mathrm{Kg}$ \\
\hline
\end{tabular}
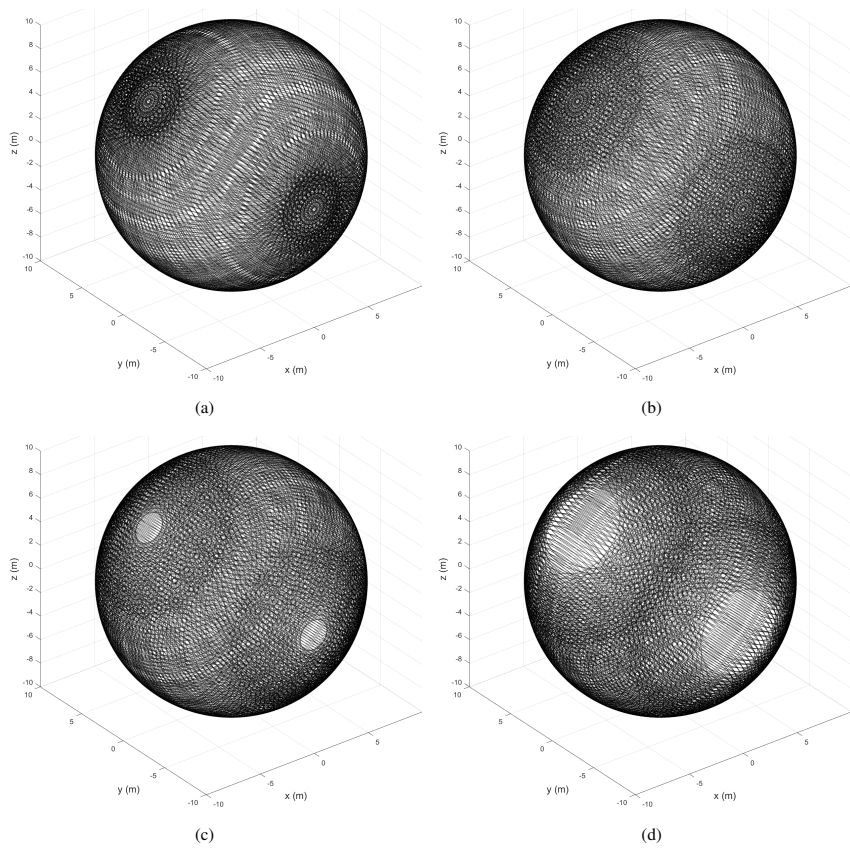

Fig. 3. Hollow sphere patterns for the Puck: (a) $\beta=0^{\circ}$, (b) $\beta=14^{\circ}$, (c) $\beta=22^{\circ}$, and (d) $\beta=35^{\circ}$.
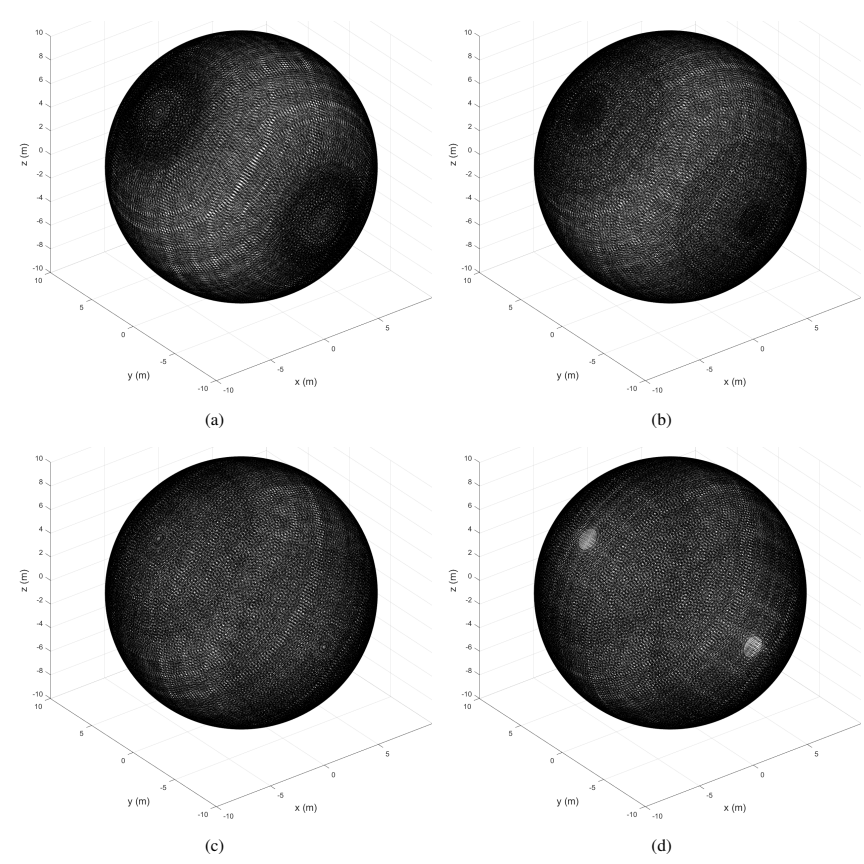

Fig. 4. Hollow sphere patterns for the HDL-32: (a) $\beta=10^{\circ}$, (b) $\beta=20^{\circ}$, (c) $\beta=31^{\circ}$, and (d) $\beta=35^{\circ}$.

rotation axis, which causes the maximum density in the focal points. The rest of the spheres in the figure indicate that with increasing values of $\beta$, density becomes lower in the focal points and unsampled stripes tend to disappear. However, the case with the highest value of $\beta$ in Fig. 3(d) (i.e., $\beta=35^{\circ}$ ) shows a significant reduction of FOV around the rotation axis. An extreme case would be $\beta=90^{\circ}$, where the rotation axis would be redundant with the Puck spinning axis and the FOV of the RMBL would be the same as that of the Puck. 
Examples of spheres computed for the HDL-32 are given in Fig. 4, where a higher density due to a doubled number of beams can be appreciated. In this case, the asymmetrical FOV of the HDL-32 implies that the highest density of the focal points is obtained when the center of the FOV is aligned with the rotation axis (i.e., $\beta=10^{\circ}$, as shown in Fig. 4(a)). Apart from that, the qualitative behavior of increasing $\beta$ is similar to the Puck case. The main difference is the resulting reduction of the FOV around the rotation axis in Fig. 4(d), which is smaller due to the wider FOV of the HDL-32.

All in all, Figs. 3-4 evidence the complexity of the measurement patterns produced by the RMBLs.

\section{K-Function Analysis and Homogeneity Optimization}

The estimation of the $K$ function in (8) is applied with discretized angle increments $\Delta \rho$ in the interval $[0, \pi]$. A value of $\Delta \rho=\pi / 151$ is suitable for the Velodyne sensors, since it allows that all bins contain a representative number of samples.

The deviation indicated by $\hat{K}(\rho)-K_{c s r}(\rho)$ using (8) and (7) with the points in the simulated RMBL spheres allows a spatial distribution analysis of sensor measurements for the whole range of normalized great circle distances $\rho$.

The curves computed for the RMBL based on a Puck sensor for configurations from $-35^{\circ}$ to $35^{\circ}$ with $1^{\circ}$ increments are presented in Fig. 5. Due to the symmetric FOV of the Puck, all curves represent both $\beta$ and $-\beta$. The configuration with $\beta=0^{\circ}$, emphasized as a bold red line in the figure, has the highest deviation from CSR. In this case, clustering is maximum around $\rho=\pi / 4 \mathrm{rad}$ and dispersion (i.e., negative values in the curve) appears when great circle distances become larger. This result is explained by the concentration of measurements in focal points about the roll axis. On the other hand, pronounced pitching of the Puck (e.g., $\beta= \pm 35^{\circ}$ ), which yields unsampled regions in the poles, causes that the largest mean number of neighbors

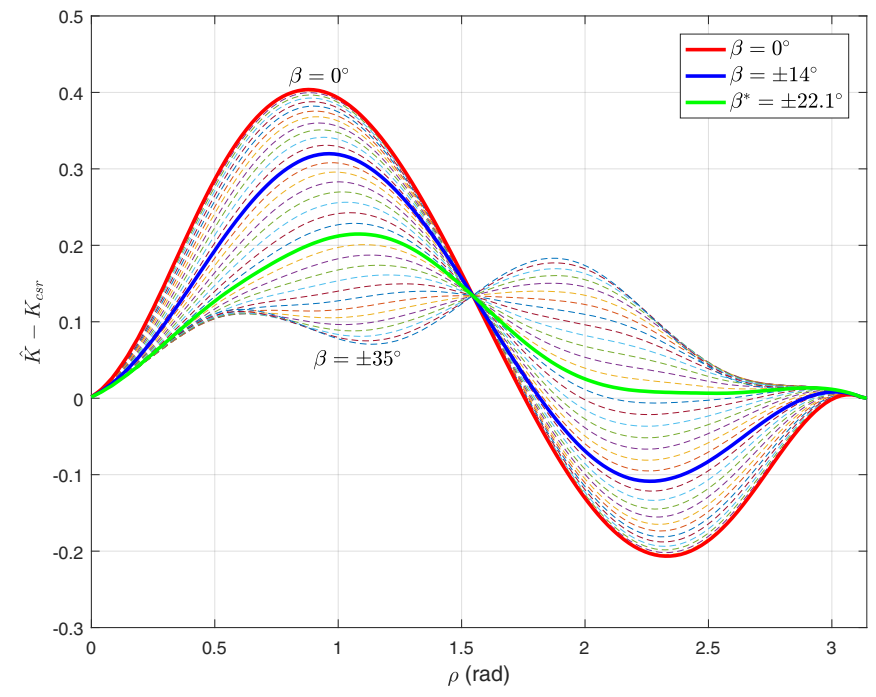

Fig. 5. Puck-based RMBL: $\hat{K}-K_{c s r}$ for $\beta$ from $-35^{\circ}$ to $35^{\circ}$ with $1^{\circ}$ increments. Bold lines indicate $\beta=0^{\circ}$ (red), $\beta= \pm 14^{\circ}$ (blue), and $\beta^{*}= \pm 22.1^{\circ}$ (green). appears within distances around $\rho=1.95 \mathrm{rad}$. In this case, the curve is always positive, meaning that there is some degree of clustering for all possible great circle distances.

The intermediate pitch value $\beta= \pm 14^{\circ}$ proposed by Neumann et al. [6] makes that the lowest beam of the Puck's FOV is almost aligned with the roll axis, which avoids the appearance of unsampled regions (i.e., it maintains the optimal FOV) while reducing the data density in the focal points, as seen in Fig. 3(b). The spatial analysis curve for this value has been emphasized as a bold blue line in Fig. 5 . The shape of this curve is closer to CSR than $\beta=0^{\circ}$, but significant clustering and dispersion appear at similar ranges of $\rho$ distances.

The computation of index $\eta$ in (9) for different pitch inclinations of a Velodyne Puck is summarized in Fig. 6, where $\beta=0^{\circ}$ reaches the highest, i.e., most inhomogeneous, value $(\eta=0.576)$. This value is clearly improved by $\beta= \pm 14^{\circ}$, with $\eta=0.406$. The configuration with the optimum pitch angle $\beta^{*}$ (with the minimum $\eta=0.267$ ) is $\beta^{*}= \pm 22.1^{\circ}$. From a qualitative standpoint, Fig. 3(c) corroborates that even if small unsampled regions appear at the poles, the overall homogeneity of this configuration is quite good, with a larger spread of focal areas around the poles and diffumination of the stripes of unsampled spots. Increasing pitch inclination above the optima reduces the FOV at the poles (Fig. 3(d)), which causes a slight increase of $\eta$, as appreciated on both extremes of Fig. 6. The spatial analysis curve for $\beta^{*}$ has been highlighted as a bold green line in Fig. 5. This distribution avoids dispersion and has a moderate clustering, which is maximum around $\rho=1.15$. Furthermore, this optimal configuration is very close to CSR, with practically no clustering and no dispersion, for normalized great circle distances over $\rho \approx 2$.

The corresponding spatial analysis curves for a RMBL based on the Velodyne HDL-32 are given in Fig. 7. The major differences with the Puck are due to its asymmetric

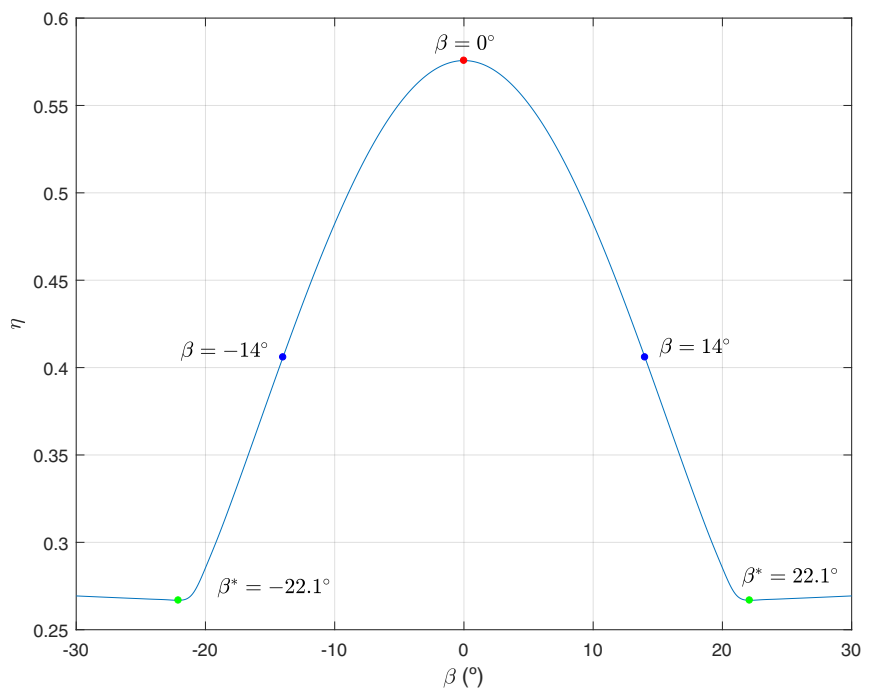

Fig. 6. Puck-based RMBL: Representation of the homogeneity index $\eta$ for different $\beta$ angles. 


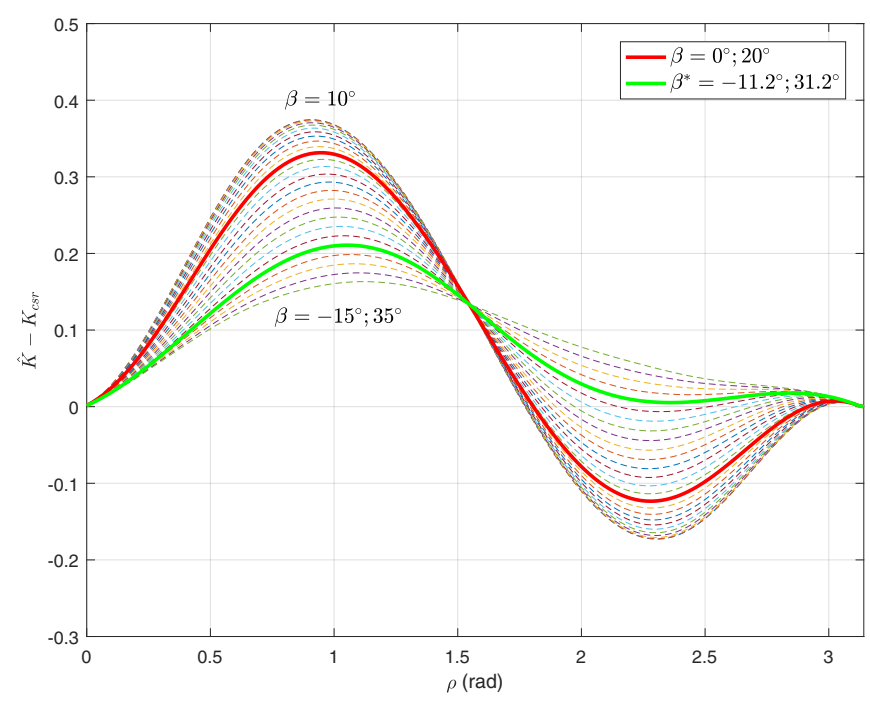

Fig. 7. HDL-32-based RMBL: $\hat{K}-K_{c s r}$ for $\beta$ from $-15^{\circ}$ to $35^{\circ}$ with $1^{\circ}$ increments. Bold lines indicate $\beta=0^{\circ} ; \beta=+20^{\circ}$ (red), and $\beta^{*}=-11.2^{\circ} ; \beta^{*}=+31.2^{\circ}$ (green).

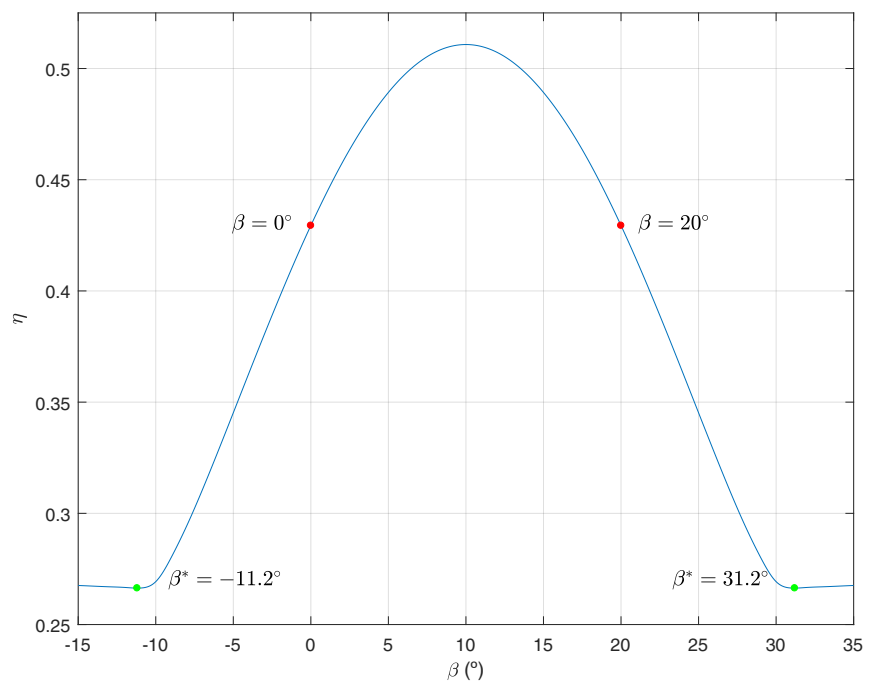

Fig. 8. HDL-32-based RMBL: Representation of the homogeneity index $\eta$ for different $\beta$ angles.

and wider FOV. The asymmetry causes an effect similar to an implicit pitch rotation. For this reason, the curve for $\beta=0^{\circ}$ is the same as for $\beta=20^{\circ}$ (emphasized as a bold red line in the figure). Thus, in this case, the most inhomogeneous curve corresponds to $\beta=10^{\circ}$, which gets the highest $\eta$ value in Fig. $8(\eta=0.511)$. For this sensor, the optima are reached for $\beta^{*}=-11.2^{\circ}$ and $\beta^{*}=31.2^{\circ}$, which achieve $\eta=0.267$. The spatial distribution curve for these pitch values (emphasized as a green bold line in Fig. 7) is very similar to the Puck optima. However, in the HDL-32, the optima does not have unsampled regions in the poles due to the wider FOV, as can be appreciated in Fig. 4(c).

\section{Effect of other RMBL Parameters}

This section discusses the effect of other operational parameters in the homogeneity of RMBL sensor data: the

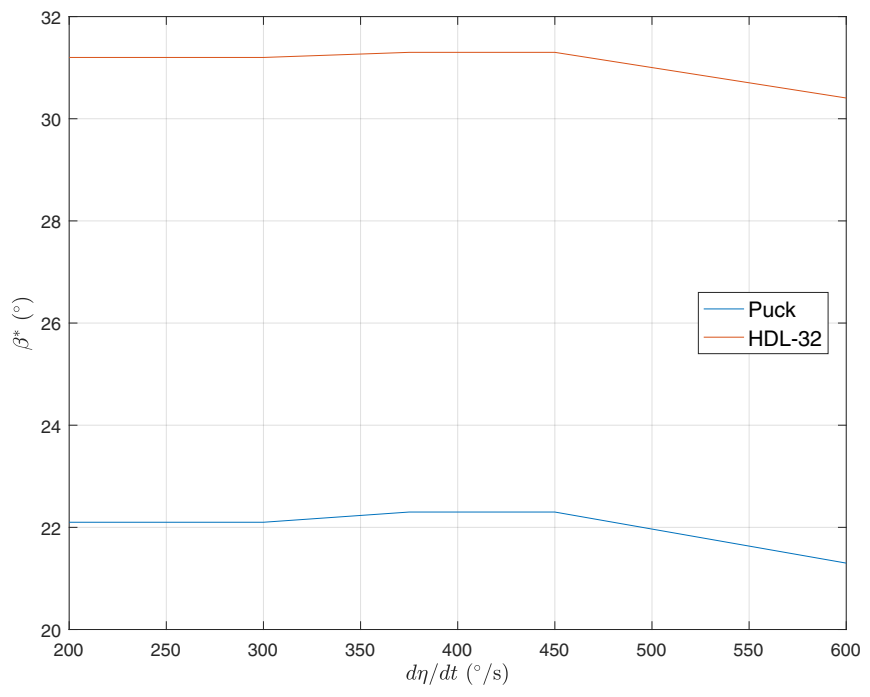

Fig. 9. Optimal Puck and HDL-32 configurations for different angular velocities.

roll speed and the distance between the optical center of the MBL and the rotation axis.

The angular velocity of $\gamma$ determines the 3D scan resolution of the RMBL. In the analysis performed above, the rotation speed was set to $d \gamma / d t=300^{\circ} / \mathrm{s}$, as in [6]. In general, the spatial distribution of scan measurements is not affected by this roll speed [5]. Nevertheless, some effect can be appreciated in the spatial analysis for the case of very fast scans (i.e., lower resolution). Fig. 9 presents the optimal Puck and HDL-32 configurations against angular velocity. Even if the variations are small, it may be of interest to consider them if the sensor is designed to operate normally with very fast rotation speeds.

As for the distance between the optical center of the MBL and the rotation axis ( $d$, as seen in Fig. 2), this has been considered null in the preceding analysis, because it is usually very short (a few centimeters) in relation with target distances. Furthermore, this assumption makes the spatial distribution analysis independent of the radius $R$ of the hollow sphere (i.e., a particular target distance). Fig. 10 shows the homogeneity index $\eta$ for different values of $d$ and $\beta$ for RMBL sensors based on both a Puck and a HDL32 rotated at $d \gamma / d t=300^{\circ} / \mathrm{s}$ inside a hollow sphere of $R=20 \mathrm{~m}$. It can be appreciated that the optimal $\beta^{*}$ is modified slightly for non-null values of $d$, as shown by the red line. Nevertheless, the optimal $\beta^{*}$ for $d=0$, shown as a dashed magenta line, remains as a quasi-optimum due to the flat shape of the homogeneity index surface near the optima.

\section{CONCLUSIONS}

Rotating a multi-beam lidar (MBL) with a servo mechanism is an effective solution to achieve high resolution and fast 3D scans with full spherical field of view (FOV), as indicated by recent published examples. However, a rotating multi-beam lidar (RMBL) yields complex scan patterns due to overlapping beams. In this paper, we have extended [5] to analyze the spatial data distribution provoked by placing 

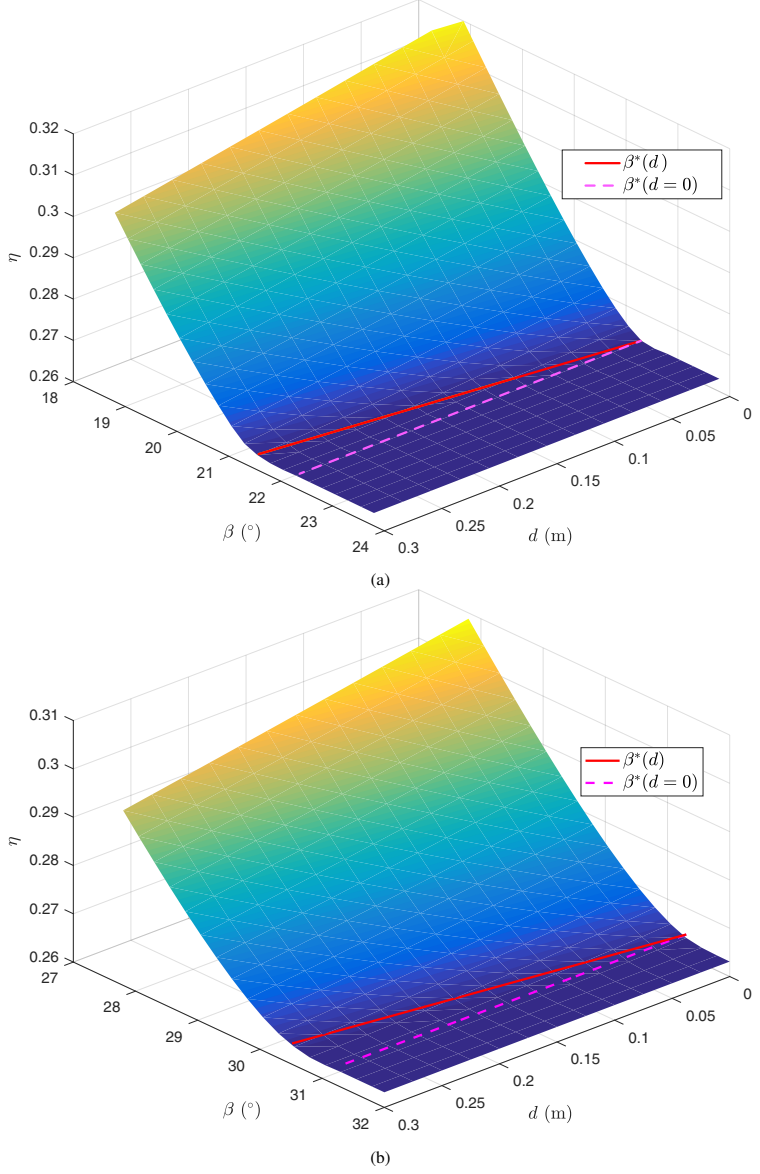

Fig. 10. (a) Puck-based RMBL and (b) HDL-32-based RMBL: Homogeneity index $\eta$ for different values of $d$ and $\beta$ and a hollow sphere with $R=20 \mathrm{~m}$. The optimum is depicted as a red line; and the quasi-optimum (i.e., assuming $d=0$ ) as a dashed magenta line.

the MBL in the RMBL mechanism with a fixed pitch angle $\beta$ with respect to the rotation axis, as proposed in [6].

Moreover, we have defined a new scalar index to assess the homogeneity of 3D data distribution on a hollow sphere by integrating the absolute difference of the $K$ function with complete spatial randomness (CSR) for all great circle distances. Using this index as a cost function, we have optimized the pitch angle that maximizes data homogeneity for RMBLs based on the Puck VLP-16 and the HDL-32.

The analysis has revealed that placing the MBL with no pitch angle, which is the most common configuration found in published works, produces inhomogeneous scans characterized by oversampled focal points and distinctive stripes of unsampled spots. The optimized configurations are $\beta= \pm 22.1^{\circ}$ for the Puck, and $\beta=-11.2^{\circ}$ or $\beta=31.2^{\circ}$ for the HDL-32. In the Puck, the optimal pitch angle causes a small unsampled region at the poles which is not present in the HDL-32 due to its larger FOV. The $K$ function for these optima shows moderate clustering but also achieves coincidence with CSR for the larger third of normalized great circle distances.

The paper has also evaluated the effects of the roll speed (i.e., scan resolution) and the distance between the optical center of the MBL and the rotation axis. These do not have a substantial effect on the optimized pitch angles, but it is interesting to note some variation of the spatial measurement distribution for very fast scans (i.e., faster than about $550^{\circ} / \mathrm{s}$ ).

These results are relevant for building customized highresolution 3D sensors in applications demanding a full FOV with a quasi-homogeneous scan resolution. Future work will be necessary to identify the effects of shadows introduced by actual rotation mechanisms. Moreover, it will be interesting to test the applicability of existing calibration methods to the rotation of multiple beams.

\section{REFERENCES}

[1] I. Bogoslavskyi and C. Stachniss. Fast range image-based segmentation of sparse 3D laser scans for online operation. In IEEE/RSJ International Conference on Intelligent Robots and Systems, pages 163-169, 2016.

[2] T. Klamt and S. Behnke. Anytime hybrid driving-stepping locomotion planning. In IEEE/RSJ International Conference on Intelligent Robots and Systems, pages 1-8, 2017.

[3] M. Leingartner, J. Maurer, A. Ferrein, and G. Steinbauer. Evaluation of sensors and mapping approaches for disasters in tunnels. J. Field Robot., 33(8):1037-1057, 2016.

[4] A. Mandow, J. Martínez, A. Reina, and J. Morales. Fast rangeindependent spherical subsampling of 3D laser scanner points and data reduction performance evaluation for scene registration. Pattern Recognition Letters, 31(11):1239-1250, 2010.

[5] J. Morales, V. Plaza-Leiva, A. Mandow, J.-A. Gomez-Ruiz, J. Serón, and A. García-Cerezo. Analysis of 3D scan measurement distribution with application to a multi-beam lidar on a rotating platform. Sensors, 18(2):395, 2018.

[6] T. Neumann, E. Dülberg, S. Schiffer, and A. Ferrein. A rotating platform for swift acquisition of dense 3D point clouds. Lecture Notes in Computer Science (including subseries Lecture Notes in Artificial Intelligence and Lecture Notes in Bioinformatics), 9834:257268, 2016.

[7] T. Neumann, A. Ferrein, S. Kallweit, and I. Scholl. Towards a mobile mapping robot for underground mines. In PRASA, RobMech and AfLaT International Joint Symposium, pages 279-284, Cape Town, South Africa, 2014.

[8] A. Pfrunder, P. V. K. Borges, A. R. Romero, G. Catt, and A. Elfes. Real-time autonomous ground vehicle navigation in heterogeneous environments using a 3D lidar. In IEEE/RSJ International Conference on Intelligent Robots and Systems, pages 2601-2608, 2017.

[9] B. D. Ripley. Modelling spatial patterns. Journal of the Royal Statistical Society. Series B (Methodological), 39(2):172-212, 1977.

[10] S. M. Robeson, A. Li, and C. Huang. Point-pattern analysis on the sphere. Spatial Statistics, 10:76-86, 2014.

[11] S. Schubert, P. Neubert, and P. Protzel. How to build and customize a high-resolution 3D laserscanner using off-the-shelf components. Lecture Notes in Computer Science (including subseries Lecture Notes in Artificial Intelligence and Lecture Notes in Bioinformatics), 9716:314 326, 2016.

[12] A. U. B. Shamsudin, N. Mizuno, J. Fujita, K. Ohno, R. Hamada, T. Westfechtel, S. Tadokoro, and H. Amano. Evaluation of LIDAR and GPS based SLAM on fire disaster in petrochemical complexes. In IEEE International Symposium on Safety, Security and Rescue Robotics, pages 48-54, 2017.

[13] M. Tonini and A. Abellan. Rockfall detection from terrestrial lidar point clouds: A clustering approach using R. Journal of Spatial Information Science, 8(1):95-110, 2014.

[14] A. Tran. Velodyne slashes the price in half of its most popular lidar sensor. Automotive Industries AI, 198(1), 2018.

[15] Velodyne LIDAR, Inc. Datasheets. In http://velodynelidar.com/docs/datasheet/, accessed on February 2018, Morgan Hill, CA, USA, 2018

[16] O. Wulf and B. Wagner. Fast 3D Scanning Methods for Laser Measurement Systems. In Proceedings of the International Conference on Control Systems and Computer Science, volume 1, pages 312-317, Bucharest, Romania, 2003. 